\section{Métabolisme et cycle cellulaire, deux processus interconnectés chez les bactéries}

Régis Hallez

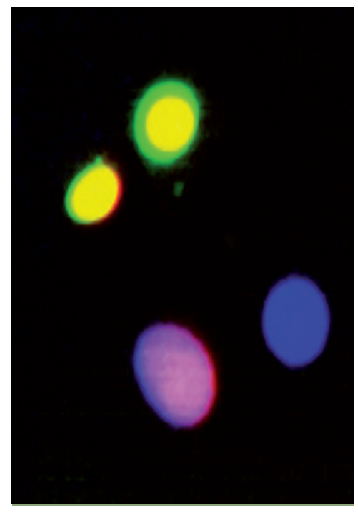

Bacterial Cell cycle and Development $(B C C D)$, unité de recherche en biologie des micro-organismes (URBM), université de Namur (UNamur), 61 rue de Bruxelles, 5000 Namur, Belgique.

regis.hallez@unamur.be

suivie d'une phase de synthèse d'acide désoxyribonucléique, ou ADN, (la phase $S$ ) au cours de laquelle le matériel génétique est dupliqué, puis d'une seconde phase de croissance (la phase G2), suivie finalement d'une phase (la phase M) durant laquelle (1) le matériel génétique est réparti de manière équivalente entre les deux cellules filles, et (2) la cytocinèse ${ }^{2}$ se déroule $\rightarrow$ Voir la Synthèse de $M$. Dorée, $m / s n^{\circ} 3$, [31] $(\rightarrow)$.

mars 2003, page 299

Le cycle cellulaire eucaryote se présente donc sous la forme des phases successives G1-S-G2-M (Figure 1). Chez les cellules procaryotes (qui ne présentent pas de noyau) comme les bactéries, le cycle cellulaire commence par une phase de croissance (la phase B ou Gl). Elle est suivie ensuite de la réplication d'ADN et, de manière concomitante, de la répartition du matériel génétique (la phase C ou S) pour se terminer par la cytocinèse (la phase $D$ ). On peut donc résumer le cycle cellulaire procaryote aux phases $B-C-D$ ou Gl-S-cytocinèse (Figure 1 ). Notons que certaines bactéries à croissance rapide initient un nouveau cycle de réplication d'ADN avant même d'avoir achevé le précédent, menant à un cycle cellulaire où la phase B (ou Gl) disparaît.

Bien qu'ayant des fonctions différentes, le métabolisme et le cycle cellulaire doivent nécessairement communiquer [1]. En effet, une cellule n'entamera pas une étape du cycle cellulaire qui est «énergivore » si son état métabolique ne le lui permet pas, par exemple en conditions de carences nutritives. De même, il serait délétère pour une cellule, en phase $S$, de catalyser des réactions métaboliques génératrices de dérivés réactifs de l'oxygène (ROS), par exemple les réactions de la chaîne de transport d'électrons en vue de produire de l'énergie,

\footnotetext{
${ }^{2}$ La cytocinèse, dernière étape du cycle cellulaire, correspond à la division du cytoplasme.
} 
qui pourraient endommager l'ADN en cours de synthèse. Pour éviter ces problèmes, les cellules disposent de mécanismes moléculaires connectant le cycle cellulaire au métabolisme. Bien qu'ayant un cycle cellulaire comportant des phases qui se chevauchent, les procaryotes n'ont pas dérogé à la règle. Une recherche intensive menée ces dix dernières années sur plusieurs espèces bactériennes modèles a permis de mettre en évidence différents mécanismes qui connectent métabolisme et cycle cellulaire. Nous illustrons ici plusieurs de ces exemples.

\section{Contrôle métabolique de la réplication d'ADN}

\section{Initiation de la réplication d'ADN (Mieux vaut prévenir que guérir)} L'initiation de la réplication d'ADN requiert la formation d'un complexe nucléoprotéique au niveau de l'origine de réplication unique qui, chez les bactéries, est stimulée par la forme active de la protéine DnaA (DnaA-ATP). En effet, en se liant à des séquences opératrices (les boîtes DnaA) qui sont présentes aux abords de l'origine de réplication, DnaA, liée à l'ATP, stimule l'ouverture de la double hélice d'ADN et facilite l'accès à la machinerie de réplication d'ADN appelée réplisome [2]. Réguler la quantité totale de DnaA, ou la proportion de DnaA liée à l'ATP, constitue donc un moyen efficace pour les bactéries de contrôler précisément l'initiation de la réplication d'ADN. II n'est donc pas surprenant que bon nombre d'entre elles possèdent des mécanismes régulant la synthèse ou l'activité de DnaA en fonction des nutriments disponibles.

C'est le cas notamment de la $\gamma$-protéobactérie ${ }^{3}$ Escherichia coli. Chez cette bactérie, la transcription du gène dnaA est inhibée lors d'une carence en acides aminés, ce qui a pour conséquence d'empêcher l'initiation de la réplication d'ADN. Cette inhibition de la transcription est due à la production d'une «alarmone », la guanosine tétra-/ penta-phosphate ou ( $p$ )ppGpp, à la suite d'une carence nutritive [3]. Cette molécule de signalisation est en effet capable de lier l'ARN polymérase (ARNP) et de modifier le programme de transcription de la cellule [4-6], en l'empêchant de transcrire notamment le gène dnaA [3] (Figure 2).

Chez l' $\alpha$-protéobactérie Caulobacter crescentus, c'est la stabilité de la protéine DnaA qui est placée sous contrôle du (p)ppGpp (Figure 2). $\varepsilon n$ effet, une carence nutritive active la synthèse de l'alarmone par l'unique (p)ppGpp synthétase SpoT [7-9], ce qui stimule, entre autres, la dégradation protéolytique de DnaA par une protéase et, de ce fait, empêche d'initier la réplication d'ADN $[8,10]$. À ce jour, nous ne savons pas comment le ( $p$ )ppGpp module la protéolyse de DnaA. L'abondance de DnaA est également régulée en fonction de la disponibilité en nutriments, et ce, d'une manière indépendante du ( $p$ ) ppGpp [11]. Cependant, le mécanisme moléculaire utilisé, qui cible la traduction de DnaA, reste inconnu à ce jour.

${ }^{3}$ Les protéobactéries sont Gram négatif. Elles possèdent une paroi cellulaire riche en lipopolysaccharides et pauvre en peptidoglycanes. Elles mesurent entre 0,2 et $10 \mu \mathrm{m}$ de long, et peuvent arborer de nombreuses morphologies différentes (en coque, en bâtonnet, en bâtonnet courbe, en spirale, etc.). La plupart d'entre elles possèdent un flagelle, ce qui leur permet de se déplacer dans le milieu. Ces organismes sont classés en cinq groupes sur la base de l'analyse du gène de l'ARN ribosomique $165: \alpha, \beta, \gamma, \delta, \varepsilon$.

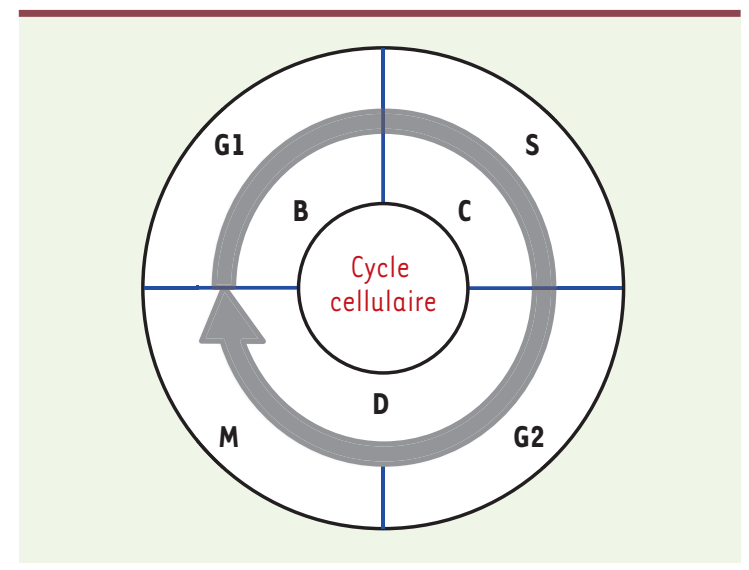

Figure 1. Représentation schématique du cycle cellulaire. Le cycle cellulaire eucaryote est composé des phases G1, S, G2 et $M$ alors que le cycle cellulaire procaryote se compose des phases $B(G 1), C(S)$ et $D$ (cytocinèse).

\section{Élongation de la réplication d'ADN} ou ségrégation (Mieux vaut tard que jamais)

Empêcher l'initiation de la réplication d'ADN en condition de carences nutritives n'est pas la seule façon de coordonner le métabolisme et la réplication d'ADN. Par exemple, chez la bactérie firmicute Bacillus subtilis, le (p)ppGpp produit lors de carences, se lie à l'ADN primase (DnaG, un composant essentiel du réplisome) en cours d'élongation de la réplication d'ADN (Figure 2). La liaison de l'alarmone à DnaG a pour conséquence d'arrêter la fourche de réplication en cours sans décrocher le réplisome de l'ADN [12].

L'étape finale de la réplication d'ADN, qui consiste à ségréger les zones de terminaison de la réplication (appelées chromosomal termini), dernière partie du chromosome à être répliquée, est également soumise à un contrôle métabolique chez les bactéries. En effet, les bactéries $\varepsilon$. coli ayant déjà initié la réplication d'ADN sont capables de réagir à une production de $(p)$ ppGpp en inhibant la ségrégation des chromosomes répliqués. Ce mécanisme implique la protéine SeqA et I'ADN méthyltransférase Dam [13]. SeqA est une protéine qui lie l'ADN hémiméthylé ${ }^{4}$ au niveau de l'origine de réplication. Cette liaison, assimilée à une séquestration, empêche DnaA-ATP de stimuler un nouveau cycle de réplication d'ADN avant que le brin néo-synthétisé ne soit méthylé par Dam. SeqA contrôle également la ségrégation des chromosomes en liant d'autres locus hémiméthylés proches des zones de terminaison de la réplication. Même si le mécanisme exact doit encore

${ }^{4}$ L'ADN hémiméthylé ne porte des groupements méthyle que sur un seul des deux brins, celui en l'occurrence qui a servi de modèle pour la réplication. 


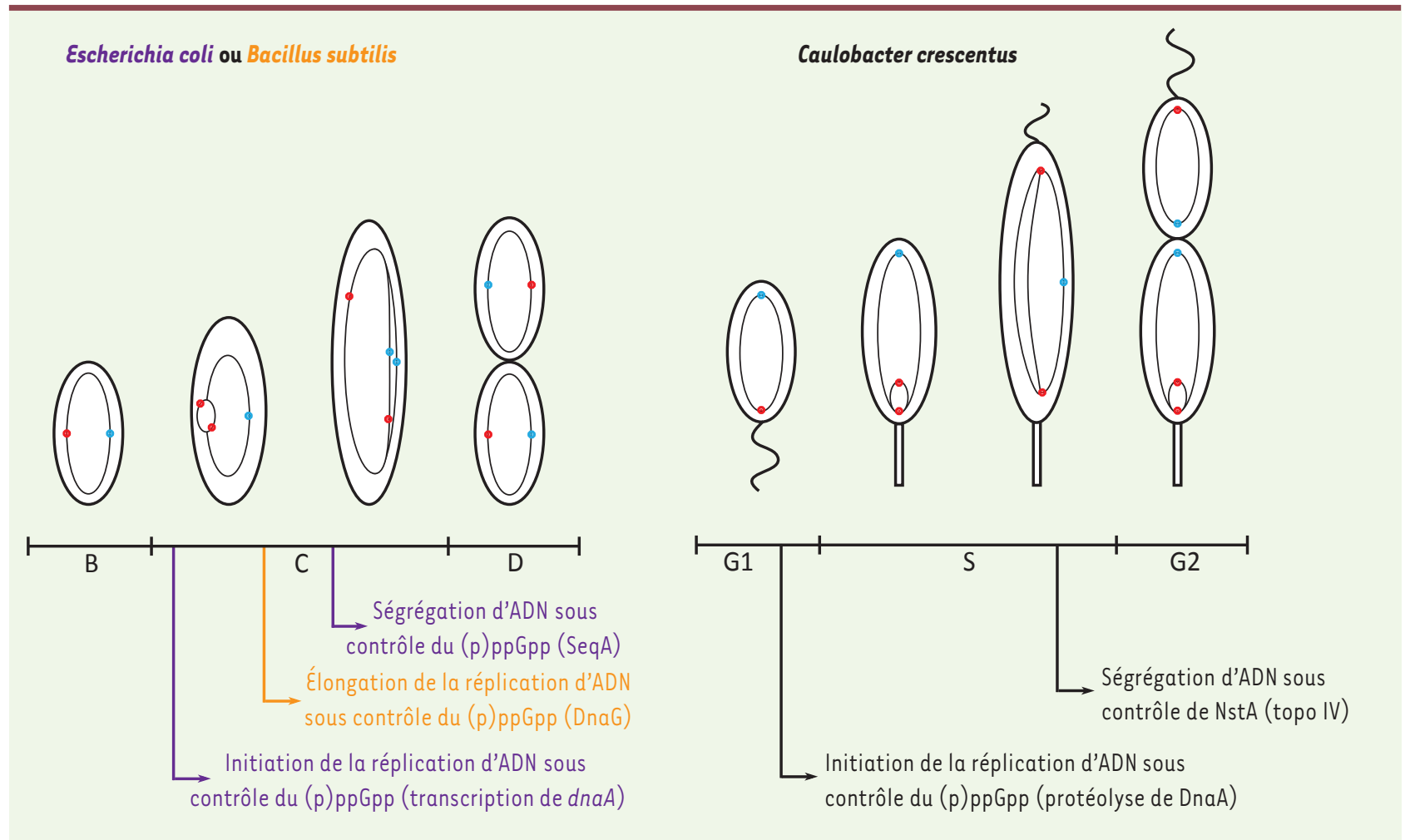

Figure 2. Contrôle métabolique de la réplication d'ADN chez les bactéries. Cycle cellulaire de la $\gamma$-protéobactérie Escherichia coli, du firmicute Bacillus subtilis ou de l' $\alpha$-protéobactérie Caulobacter crescentus. Les moments du cycle cellulaire auxquels les mécanismes décrits dans le texte interviennent sont indiqués en violet pour $\varepsilon$. coli, en orange pour $B$. subtilis et en noir pour $C$. crescentus. La (ou les) origine(s) de réplication d'ADN est(sont) représentée(s) en rouge et les zones de terminaison de la réplication d'ADN (termini) en bleu. NstA : negative switch for topo IV decatenation activity; topo IV : topo-isomérase IV.

être identifié, l'alarmone semble contrôler la ségrégation chromosomique en influençant la liaison de SeqA aux sites de liaison proches des termini [13] (Figure 2).

Un autre exemple de communication entre le métabolisme et la ségrégation des chromosomes a été décrit chez $C$. crescentus. II s'agit de la régulation de l'activité de la topo-isomérase IV (topo IV) par un inhibiteur sensible à l'état d'oxydoréduction de la bactérie [14]. La topo IV est une enzyme qui résout les problèmes topologiques engendrés par le processus de réplication d'ADN. Plus particulièrement, cette enzyme dénoue, en fin de cycle de réplication, les chromosomes «entremêlés» et, de ce fait, favorise une ségrégation optimale des chromosomes répliqués. De manière surprenante, la topo IV est présente tout au long du cycle cellulaire alors que son activité n'est requise qu'une fois le chromosome dupliqué, ce qui suggère que son activité serait inhibée au cours de la phase $S$. Chez $C$. crescentus, un inhibiteur (NstA, negative switch for topo IV decatenation activity) restreint l'activité de la topo IV en fin de cycle [14]. Plus précisément, NstA n'est actif (en tant qu'inhibiteur de la topo IV) que dans un environnement oxydé. Étant donné que l'état redox (oxydoréduction) varie au cours du cycle cellulaire chez $C$. crescentus, avec un environnement réduit en phases G1 et G2 et oxydé en phase $S$, l'activité de NstA est ainsi restreinte à la phase $S$. Autrement dit, l'inhibiteur NstA est inactivé en fin de phase S (lorsque l'environnement intracellulaire devient réducteur), ce qui libère l'activité de la topo IV [14] (Figure 2). Non seulement ce système permet de synchroniser les différentes étapes de la réplication d'ADN, mais il constitue également une connexion supplémentaire entre le métabolisme et le cycle cellulaire. $\varepsilon n$ effet, un problème métabolique survenant en cours de réplication et empêchant la réduction du cytoplasme maintiendra la topo IV inactive et empêchera la ségrégation des chromosomes.

\section{Contrôle métabolique de la cytocinèse}

\section{Adaptation de la taille cellulaire en fonction}

de la disponibilité en nutriments

(Plus tu manges, plus tu es grand)

Les bactéries à croissance rapide sont connues depuis plus de 50 ans pour être capables d'adapter leur taille cellulaire en fonction de leur vitesse de croissance (donc de la disponibilité en nutriments). Par exemple, les bactéries $\varepsilon$. coli, B. subtilis, Salmonella typhimurium et Pseudomonas aeruginosa ont une taille cellulaire 
plus importante lorsqu'elles sont cultivées en milieu riche, c'est-àdire lorsqu'elles ont une vitesse de croissance plus élevée [15-18]. L'augmentation du volume cellulaire associée à cette augmentation de taille permettrait aux bactéries de supporter une réplication multiple de leurs chromosomes. En effet, toutes ces espèces bactériennes présentent la particularité, lorsqu'elles sont cultivées en milieu riche, d'initier plusieurs fois la réplication de leur matériel génétique avant de se diviser. Cette simple corrélation est restée énigmatique pendant près de 40 ans avant qu'un mécanisme moléculaire ne soit identifié chez $B$. subtilis grâce à un crible génétique. Ce crible a consisté à chercher des mutants incapables d'adapter correctement leur taille cellulaire en condition de culture en milieu riche, en d'autres termes des mutants présentant une taille cellulaire moyenne plus petite que la souche sauvage pour une vitesse de croissance élevée. Ce crible a permis de mettre en évidence l'implication de la glucosyltransférase UgtP [19] (Figure 3). Cette enzyme participe à la biosynthèse de glucolipides, précurseurs de constituants essentiels des parois bactériennes. Outre son rôle métabolique, UgtP interagit avec la GTPase FtsZ, une interaction favorisée en présence d'une forte concentration en uridine diphosphate-glucose (UDP-glucose, le substrat d'UgtP), ce qui est le cas en milieu riche. FtsZ, homologue lointain de la tubuline eucaryote, est impliqué chez la très grande majorité des bactéries dans le processus de cytocinèse $[20,21]$. La liaison de la guanosine triphosphate (GTP) à FtsZ stimule en effet sa polymérisation en protofilaments qui interagissent latéralement entre eux pour former une structure plus complexe prenant la forme d'un anneau circonférentiel (anneau Z), au centre de la cellule et en contact étroit avec la membrane. L'anneau Z joue deux rôles essentiels dans le processus de cytocinèse. II sert en effet d'échafaudage moléculaire en recrutant toutes les protéines nécessaires à la cytocinèse. Mais l'activité GTPase, intrinsèque de FtsZ, fournit, une fois stimulée en fin de cycle cellulaire, l'énergie nécessaire au rapprochement des membranes et des parois ainsi qu'à leur fusion lors de la séparation finale des deux cellules filles [22]. Étant donné le rôle central de FtsZ dans le processus de cytocinèse, il n'est pas surprenant que les bactéries disposent de mécanismes régulant l'abondance, ou l'activité, de FtsZ pour coordonner leur division et leur métabolisme. Dans l'exemple cité ci-dessus, I'interaction d'UgtP et de FtsZ limite les monomères de FtsZ disponibles pour la polymérisation. $\varepsilon$ n conséquence, la formation de l'anneau $Z$ et la cytocinèse qui en dépend sont retardées dans des cellules mères de $B$. subtilis qui sont cultivées en milieu riche (forte concentration intracellulaire en UDPglucose), pour finalement donner naissance à des cellules filles de plus grande taille $[19,23]$.

Ce mécanisme efficace existe également chez $\varepsilon$. coli même s'il engage d'autres protéines. $\varepsilon$ n effet, cette bactérie utilise une autre glucosyltransférase, $\mathrm{OpgH}$, pour coordonner le métabolisme et la cytocinèse [24]. Tout comme UgtP chez B. subtilis, 0 pgH chez $\varepsilon$. coli est non seulement impliquée dans la biosynthèse de constituants de la paroi (les glucanes périplasmiques), mais interfère également avec la formation de l'anneau Z en interagissant avec FtsZ, avec pour conséquence la séquestration d'une partie des monomères de FtsZ qui sont, dès lors, indisponibles à la polymérisation [24] (Figure 3). L'interaction entre
$\mathrm{OpgH}$ et FtsZ requiert également I'UDP-glucose, le substrat de $0 \mathrm{pgH}$. UgtP et $0 \mathrm{pgH}$ constituent un exemple typique d'évolution convergente. Ces deux enzymes ne sont pas homologues et elles catalysent des réactions différentes. Mais elles contrôlent toutes deux la cytocinèse en interagissant avec FtsZ, et ce, uniquement lorsque la concentration en UDP-glucose est élevée.

Chez $B$. subtilis, un autre système permet de connecter le métabolisme à la cytocinèse. Cette connexion a été révélée grâce à un autre crible génétique qui a consisté à sélectionner des mutants capables de restaurer la viabilité d'un mutant thermosensible de $f t s Z\left(f t s Z_{t s 1}{ }^{5}\right)$ à haute température. En effet, des mutations inactivant des gènes impliqués dans le processus de glycolyse restaurent la capacité du mutant $f t s Z_{t s 1}$ à croître à une température de $37^{\circ} \mathrm{C}$ [25]. Même si le mécanisme précis reste inconnu, le pyruvate et la pyruvate déshydrogénase, complexe enzymatique transformant le pyruvate en acétyl-coenzyme $A$ (acétyl-CoA), semblent être impliqués [25].

\section{Coordination de la cytocinèse avec le métabolisme (Tout vient à point à qui sait attendre)}

Contrairement aux bactéries à croissance rapide, $C$. crescentus n'adapte pas sa taille cellulaire en fonction de la disponibilité en nutriments. Qu'elle soit cultivée en milieu synthétique (pauvre en nutriments) dans lequel elle doit synthétiser tout ce dont elle a besoin pour croître à partir du glucose comme seule source de carbone, ou qu'elle croisse en milieu complexe (riche en nutiments) contenant de nombreux nutriments directement assimilables, la bactérie $C$. crescentus présente une taille similaire [26]. Le cycle de C. crescentus est un peu particulier. Elle se divise de manière asymétrique pour donner naissance à une grande cellule pédonculée qui est attachée au substrat grâce à une substance collante (un exopolysaccharide appelé holdfast), et une petite cellule flagellée capable de nager dans son environnement aquatique (Figures 2 et 3 ). Alors que la cellule pédonculée ré-initie un nouveau cycle de réplication (phase $S$ ) immédiatement après la cytocinèse, la cellule flagellée passe obligatoirement par une phase Gl avant d'entrer en phase $\mathrm{S}$. De manière concomitante à l'entrée en phase $S$, la cellule flagellée se différencie alors en cellule pédonculée, notamment en éjectant son flagelle polaire et en synthétisant un nouveau pédoncule au même pôle.

Bien que sa taille cellulaire ne soit pas modifiée en fonction de la disponibilité en nutriments, C. crescentus utilise néanmoins des systèmes pour connecter son métabolisme à la division cellulaire. Un de ces

\footnotetext{
${ }^{5}$ Le mutant thermosensible $\mathrm{fts} \mathrm{Z}_{\mathrm{ts}}$ est incapable de se diviser à $37^{\circ} \mathrm{C}$.
} 


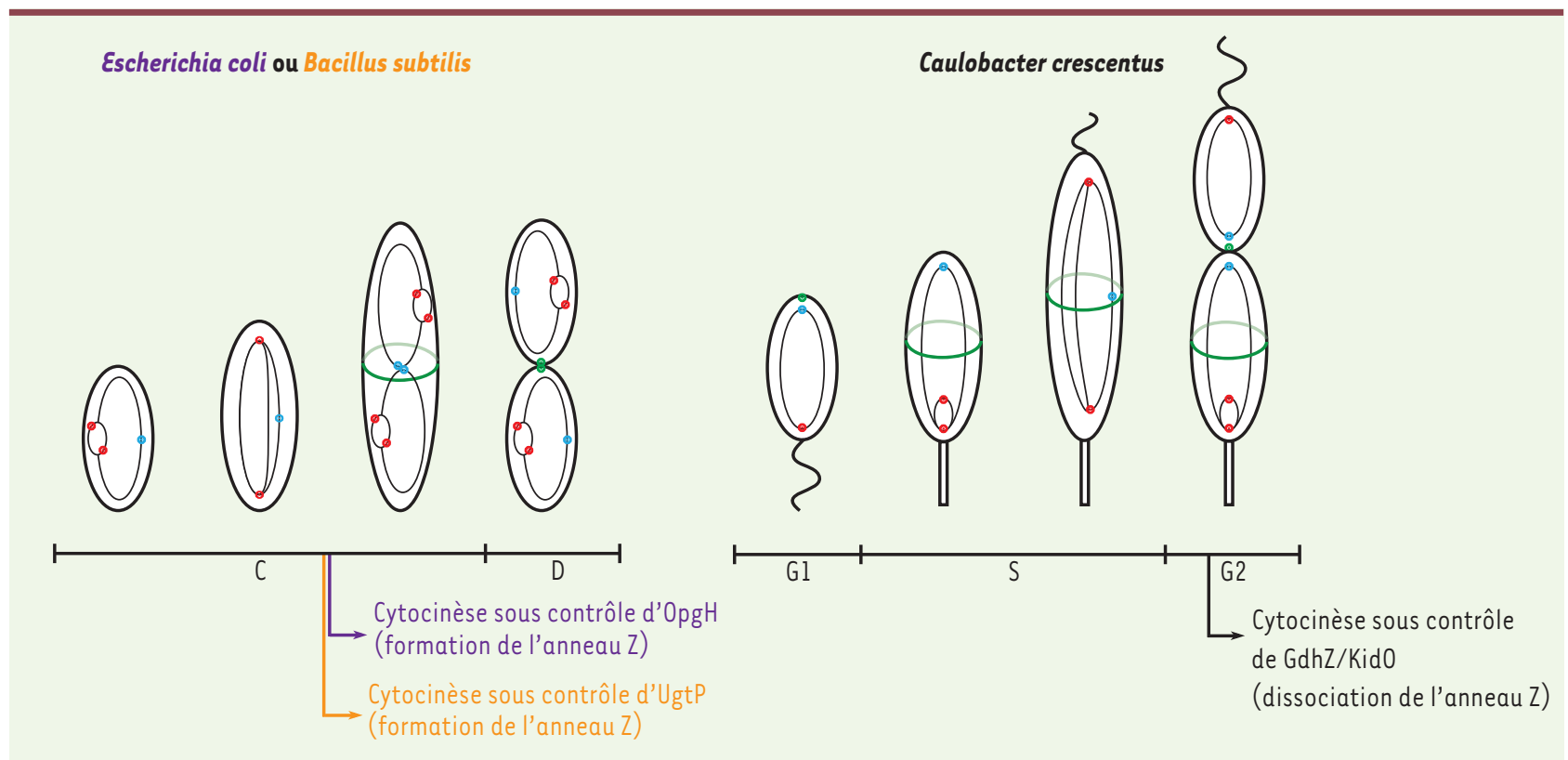

Figure 3. Contrôle métabolique de la cytocinèse chez les bactéries. Cycle cellulaire de la $\gamma$-protéobactérie Escherichia coli, du firmicute Bacillus subtilis et de l' $\alpha$-protéobactérie Caulobacter crescentus. Les moments du cycle cellulaire auxquels les mécanismes décrits dans le texte interviennent sont indiqués en violet pour $\varepsilon$. coli, en orange pour $B$. subtilis et en noir pour $C$. crescentus. La ou les origine(s) de réplication d'ADN est(sont) représentée(s) en rouge et les zones de terminaison de la réplication d’ADN (termini) en bleu. L'anneau Z, constitué de la protéine FtsZ, est représenté en vert. Notons que la réplication multiple du chromosome unique de $\varepsilon$. coli et $B$. subtilis a pour conséquence de faire disparaître la phase pré-réplicative $B(\mathrm{Gl})$. Notons également que la phase de cytocinèse (phase $D$ chez $\varepsilon$. coli et $B$. subtilis) est appelée pour des raisons historiques phase $\mathrm{G} 2$ chez $C$. crescentus.

systèmes comprend deux protéines, GdhZ et Kid0 (Figure 3). GdhZ est une glutamate déshydrogénase dépendante de la coenzyme nicotinamide adénine dinucléotide (NAD), qui catabolise le glutamate en $\alpha$-cétoglutarate et en ammonium tout en réduisant le $\mathrm{NAD}^{+}$en $\mathrm{NADH}$ $+\mathrm{H}^{+}$. KidO, quant à elle, est une oxydoréductase inactive mais qui est toujours capable de lier le $\operatorname{NAD}(H)$. Ces deux protéines peuvent interagir avec FtsZ et interférer avec la stabilité de l'anneau $Z$ en utilisant des mécanismes qui sont différents mais complémentaires [27, 28]. $\varepsilon n$ effet, GdhZ réduit la taille des protofilaments de FtsZ en stimulant son activité GTPase, alors que KidO déstabilise les interactions latérales entre protofilaments au sein de l'anneau $Z$ [27]. Cette régulation nécessite la liaison d'un substrat à GdhZ et à KidO. Le $N A D^{+}$, ou le glutamate, sont donc requis pour GdhZ et le NADH, pour KidO. L'abondance de ces deux protéines oscille au cours du cycle cellulaire pour atteindre une concentration élevée au moment de la cytocinèse. La présence concomitante de GdhZ et de KidO permet à la bactérie de stimuler la désintégration rapide de l'anneau, une étape indispensable au bon déroulement de la cytocinèse. La liaison obligatoire des substrats permet dès lors à $C$. crescentus de ne stimuler la division cellulaire que si l'apport nutritif est suffisant [27]. À l'inverse, une carence carbonée pourrait retarder la cytocinèse et le largage de la cellule flagellée. Ce mécanisme éviterait la naissance d'une cellule flagellée dans un environnement pauvre en carbone. Le contrôle de la cytocinèse par GdhZ semble être conservé au sein d'autres $\alpha$-protéobactéries [29].

\section{Conclusions}

Loin d'être exhaustifs, ces quelques exemples illustrent les connexions identifiées ces dernières années entre le métabolisme et le cycle cellulaire chez les bactéries. Longtemps étudié comme de simples réactions chimiques indépendantes les unes des autres, le métabolisme dévoile maintenant une nouvelle facette. Certaines enzymes peuvent posséder deux fonctions distinctes qui sont complémentaires: une fonction de catalyse chimique et une de contrôle, permettant de communiquer l'état métabolique de la cellule à d'autres processus. Ces enzymes, appelées «moonlighting ${ }^{6}$ » pour leur seconde fonction bien souvent masquée par la première, sont loin d'être minoritaires puisque la plupart des enzymes glycolytiques et du cycle de Krebs semblent comporter une seconde fonction [30]. Ces recherches fondamentales sur les bactéries pourraient également inspirer les recherches sur les cellules tumorales. D'une part, les cellules tumorales sont en effet caractérisées par un cycle cellulaire débridé et un métabolisme fortement altéré. D'autre part, les

\footnotetext{
${ }^{6}$ «Travail au noir ».
} 
bactéries et les cellules humaines utilisent les mêmes métabolites. Une connexion entre un métabolite et une des étapes du cycle cellulaire pourrait ainsi, dès lors, être conservée de la bactérie à l'homme, même si les mécanismes moléculaires impliqués diffèrent. $\diamond$

\section{LIENS D'INTÉRÊT}

Les auteurs déclarent n'avoir aucun lien d'intérêt concernant les données publiées dans cet article.

\section{SUMMARY}

Metabolism and cell cycle, two interconnected processes in bacteria To increase chances for a cell to survive in its natural environment, metabolism and cell cycle necessarily have to be interconnected. Indeed, cells need, on the one hand, to check their metabolic status before initiating a cell cycle step often energy-consuming, and, on the other hand, to complete some cell cycle steps before modifying their metabolism. Because bacteria do not differ from the rules, an increasing number of examples of connection between metabolism and cell cycle emerged these last years. Identifying metabolic enzymes as messengers coordinating metabolism and cell cycle allows the addition of another dimension to metabolic maps. Likewise, the very high conservation of these metabolic maps, from bacteria to human, allows inspiring research on tumor cells that are known to have an unrestrained cell cycle and a voracious appetite. $\diamond$

\section{RÉFÉRENCES}

1. Cai L, Tu BP. Driving the cell cycle through metabolism. Annu Rev Cell Dev Biol $2012 ; 28: 59-87$.

2. Magnan D, Bates D. Regulation of DNA replication initiation by chromosome structure. J Bacteriol $2015 ; 197: 3370-7$.

3. Chiaramello AE, Zyskind JW. Coupling of DNA replication to growth rate in Escherichia coli: a possible role for guanosine tetraphosphate. J Bacteriol $1990 ; 172: 2013-9$.

4. Artsimovitch I, Patlan V, Sekine $S$, et al. Structural basis for transcription regulation by alarmone ppGpp. Cell $2004 ; 117: 299-310$

5. Ross $W$, Vrentas $C E$, Sanchez-Vazquez P, et al. The magic spot: a ppGpp binding site on $\varepsilon$. coli RNA polymerase responsible for regulation of transcription initiation. Mol Cell $2013 ; 50: 420-9$.

6. Zuo $Y$, Wang $Y$, Steitz TA. The mechanism of $\varepsilon$. coli RNA polymerase regulation by $\mathrm{ppGpp}$ is suggested by the structure of their complex. Mol Cell $2013 ; 50: 430-6$.

7. Boutte CC, Crosson S. The complex logic of stringent response regulation in Caulobacter crescentus: starvation signalling in an oligotrophic environment. Mol Microbiol $2011 ; 80$ : 695-714.

8. Lesley JA, Shapiro L. SpoT regulates DnaA stability and initiation of DNA replication in carbonstarved Caulobacter crescentus.J Bacteriol 2008 ; 190 : 6867-80.

9. Ronneau S, Petit K, De Bolle X, Hallez R. Phosphotransferase-dependent accumulation of ( $p$ ) ppGpp in response to glutamine deprivation in Caulobacter crescentus. Nat Commun 2016 ; $7: 11423$

10. Gorbatyuk B, Marczynski GT. Regulated degradation of chromosome replication proteins DnaA and CtrA in Caulobacter crescentus. Mol Microbiol 2005 ; 55 : 1233-45.
11. Leslie DJ, Heinen C, Schramm FD, et al. Nutritional control of DNA replication initiation through the proteolysis and regulated translation of DnaA. PLOS Genet $2015 ; 11$ : e1005342.

12. Wang JD, Sanders GM, Grossman AD. Nutritional control of elongation of DNA replication by (p)ppGpp. Cell $2007 ; 128: 865-75$.

13. Ferullo DJ, Lovett ST. The stringent response and cell cycle arrest in Escherichia coli. PLoS Genet $2008 ; 4$ : e1000300.

14. Narayanan S, Janakiraman B, Kumar L, Radhakrishnan SK. A cell cyclecontrolled redox switch regulates the topoisomerase IV activity. Genes Dev $2015 ; 29: 1175-87$.

15. Cooper $\mathrm{S}$, Helmstetter $C \varepsilon$. Chromosome replication and the division cycle of Escherichia coli B/r.J Mol Biol 1968 ; 31 : 519-40

16. Donachie WD, Begg KJ. Cell length, nucleoid separation, and cell division of rod-shaped and spherical cells of Escherichia coli.J Bacteriol 1989; 171 : 4633-9.

17. Sargent MG. Control of cell length in Bacillus subtilis. J Bacteriol 1975; 123 : 7-19.

18. Schaechter M, Maaloe 0, Kjeldgaard NO. Dependency on medium and temperature of cell size and chemical composition during balanced grown of Salmonella typhimurium. J Gen Microbiol 1958 ; 19 : 592-606.

19. Weart RB, Lee $A H$, Chien $A C$, et al. A metabolic sensor governing cell size in bacteria. Cell 2007 ; $130: 335-47$.

20. Errington J, Daniel RA, Scheffers DJ. Cytokinesis in bacteria. Microbiol Mol Biol Rev $2003 ; 67: 52-65$.

21. Margolin W. FtsZ and the division of prokaryotic cells and organelles. Nat Rev Mol Cell Biol $2005 ; 6: 862-71$.

22. Erickson HP, Anderson DE, Osawa M. FtsZ in bacterial cytokinesis: cytoskeleton and force generator all in one. Microbiol Mol Biol Rev 2010 ; 74 : 504-28.

23. Chien AC, Zareh SK, Wang YM, Levin PA. Changes in the oligomerization potential of the division inhibitor UgtP co-ordinate Bacillus subtilis cell size with nutrient availability. Mol Microbiol 2012 ; 86 : 594-610.

24. Hill NS, Buske PJ, Shi Y, Levin PA. A moonlighting enzyme links Escherichia coli cell size with central metabolism. PLoS Genet 2013 ; 9 : el003663.

25. Monahan LG, Hajduk IV, Blaber SP, et al. Coordinating bacterial cell division with nutrient availability: a role for glycolysis. MBio 2014 ; 5 : e00935-14.

26. Campos M, Surovtsev IV, Kato $S$, et al. A constant size extension drives bacterial cell size homeostasis. Cell 2014 ; 159 : 1433-46.

27. Beaufay F, Coppine J, Mayard A, et al. A NAD-dependent glutamate dehydrogenase coordinates metabolism with cell division in Caulobacter crescentus. EMBOJ $2015 ; 34: 1786-800$.

28. Radhakrishnan SK, Pritchard S, Viollier PH. Coupling prokaryotic cell fate and division control with a bifunctional and oscillating oxidoreductase homolog. Dev Cell 2010 ; 18 : 90-101.

29. Beaufay F, De Bolle X, Hallez R. Metabolic control of cell division in alphaproteobacteria by a NAD-dependent glutamate dehydrogenase. Commun Integr Biol 2016 ; 9 : el125052.

30. Huberts DH, van der Klei IJ. Moonlighting proteins: an intriguing mode of multitasking. Biochim Biophys Acta $2010 ; 1803$ : 520-5.

31. Dorée M. Le déclenchement de la mitose chez les eucaryotes supérieurs. Med Sci (Paris) $2003 ; 19: 299-307$.

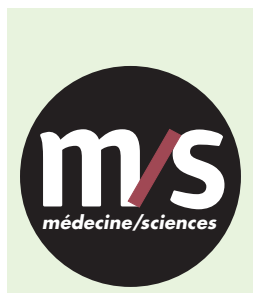

Tarifs d'abonnement $\mathrm{m} / \mathrm{s}-2016$

$>$ Grâce à $m / s$, vivez en direct les progrès des sciences biologiques et médicales

\section{TIRÉS À PART}

R. Hallez

\section{Abonnez-vous \\ à médecine/sciences}

Bulletin d'abonnement page 910 dans ce numéro de $\mathrm{m} / \mathrm{s}$

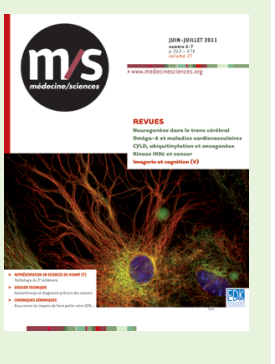

